\title{
Una propuesta de plan estratégico para el Departamento de Ciencias Médicas doctor Salvador Allende La Habana Cuba
}

\author{
María Luisa Machado Marquetti
}

\begin{abstract}
RESUMEN
En está investigación se presenta una propuesta del plan estratégico con una proyección de desarrollo en el Departamento Docente de Licenciatura en Enfermería de la Facultad de Ciencias Médicas doctor Salvador Allende Ciudad Habana, Cuba para una temporalidad del 2003 a 2007. Para la elaboración de la propuesta del plan estratégico de este departamento se aplica tecnología de corte estratégico diseñado para nivel institucional, la que ha sido viabilizada en esta investigación, con la teoría de inducción analítica, para el nivel departamental. El enfoque metodológico aplicado en esta propuesta esta conformado por tres etapas: filosófica, analítica y operativa. En la etapa filosófica: se logra declarar la esencia y razón de ser de la organización. Etapa analítica: basada en un análisis de la situación actual o diagnóstico y la etapa operativa donde se presenta un escenario prospectivo conformado por políticas, objetivos y estrategias. La direccionalidad del cambio se argumenta con la consideración de que el departamento constituye una organización abierta al aprendizaje, en la que se proyecta la transformación de la docencia, investigación y extensión universitaria soportada en el fortalecimiento de los recursos y la cooperación. Se sustenta el cambio con la presencia del factor humano que ha desarrollado un marco de compromisos y participación de todos los actores de la organización académica, además de la comunicación y motivación dentro del contexto del departamento.
\end{abstract}

Palabras clave: planeación estratégica, direccionalidad, cambio.

\section{A proposal for a strategic plan for the Department of Science Medical "Science Medical Dr. Salvador Allende" Habana Cuba}

\begin{abstract}
This research presents an approach for a strategic plan with a projection of development in the Teaching Department of Degree Nursing from the faculty of medical sciences "MD Salvador Allende" Habana City, Cuba for a temporality of the 2003-2007. For the proposal elaboration for a strategic plan of this department its applied technology for strategic court, designed for the institutional level, which has been made possible in this research, with the analytical induction theory, for the department level. The methodological approach in this proposal consisting of three stages: the philosophical, analytical, and operational. The philosophical stage declare the essence and purpose of the organization, the analytical phase is based on an analysis of the current situation or a diagnosis, and in the operational phase, where prospective scenery is formed by policies, objectives and strategies. The change directionality argues with the consideration that the department is an organization open to learning, which is projected the transformation of teaching, research and university extension, supported in the encouragement of resources and cooperation. The change is supported with the presence of the human factor that has developed a framework of commitments and involvement of all participants in the academic organization also the communication and motivation inside of the department context.
\end{abstract}

Key words: strategic planning, directionality, change.

Correspondencia: Maria Luisa Machado Marquetti. Universidad Autónoma Metropolitana Xochimilco, Calz. del Hueso 1100. Col. Villa Quietud 04960 México, D.F. Email: mariamachado5@yahoo.com.mx 
$\mathrm{L}$

as instituciones de educación superior del contexto Latinoamericano y del caribe, en los momentos actuales se encuentran sometidas a grandes retos frente a los significativos cambios que se están presentando en el contexto interno, lo que ha determinado que las mismas tienen lugar en su contexto interno, lo que ha determinado que las mismas tengan que asumir reorientación y redefinición de sus propósitos.

La educación superior cubana también esta incluída en estos nuevos escenarios que están formados por nuevos agentes de cambios y retos futuros. Lo anteriormente expuesto ha conllevado a que estas instituciones educativas tiendan a realizar cambios estratégicos importantes en sus funciones sustantivas y procesos complementarios para alcanzar una visión de futuro que sea posible de lograr por medio de la aplicación de procesos de mejoramiento y transformación desde una perspectiva más amplia y así eliminar la inmediatez, e irracionalidad en la utilización de recursos e improvisaciones, por parte de los directivos en sus actividades de gestión en estas instituciones educativas. En Cuba se observan preocupaciones por el perfeccionamiento de la dirección con relación a la instrumentación de los procesos de planificación estratégica. Las universidades médicas aún se encuentran en una fase muy incipiente en el desarrollo de sus planes estratégicos a mediano y largo plazo, los cuales se operacionalizan fundamentalmente a través de implementación de objetivos anuales, que se elaboran sobre las mismas bases, principios, métodos y técnicas de la dirección por objetivos.

La investigación que presentamos se refiere a la implementación de estratégias que conducen a un proceso de cambio; en una institución educativa esta proyectada para su realización en un departamento docente de una facultad de medicina, donde se imparte una carrera que tiene la característica de ser de reciente creación.

\section{Objetivo general}

Elaborar una propuesta de plan estratégico de desarrollo integral, que posibilite un proceso de cambio en las funciones sustantivas de la universidad en el departamento de licenciatura en enfermería.

\section{Objetivos específicos}

1. Diseñar un diagnóstico que permita identificar las principales fortalezas y debilidades que se presentan; en el desarrollo de las funciones sustantivas en el Departamento Docente de Licenciatura en Enfermería.

2. Implementar un modelo de plan estratégico en el departamento de licenciatura en enfermería.

\section{MARCO TEÓRICO}

La planificación estratégica es considerada como un proceso que permite asegurar la competitividad presente y futura de quien lo impulsa, de una organización o un territorio, analizando la propia situación interna y anticipando la evolución del entorno concretando las ideas en planes y programas de acción, definido en el tiempo y espacio formulado en términos de corte inversión y resultados.

El proceso de planificación estratégica tiene la característica de ser sistemático y secuencial por su sentido de organización y conducción operacional o administrativa que tiene lugar por etapas programadas y actúa en base a una realidad concreta, además indica que la planificación estratégica debe ser flexible para poder aprovechar el conocimiento acerca del medio ambiente.

La planificación estratégica esta basada fundamentalmente en el desarrollo de la construcción social, donde el conocimiento del proceso es de sujeto- sujeto (Flores 1992).

La planificación estratégica ha tenido también su impacto en las universidades; porque los escenarios que se presentan en estos momentos determinan la necesidad que las instituciones de educación superior incluyan en los marcos de su gestión una cultura de planificación, caracterizada por un pensamiento estratégico que conlleve a las instituciones educativas a que se preparen de antemano para conocer hacia donde va la institución en el futuro y como alcanzar esa imagen futura deseada acorde a las exigencias que se promueven para este siglo.

\section{MATERIAL Y MÉTODOS}

Se realizó una investigación cualitativa y prospectiva, utilizando un modelo de planeación estratégica, mediante la teoría de inducción analítica como parte de la investigación cualitativa. El modelo que se aplicó fue diseñado originalmente en la Universidad, JM Saracho, Tarija Bolivia, en la cual se vio la transformación de esa universidad en cuanto funciones sustantivas.

Se aplicó el instrumento de la Matriz Foda, (Znacki) 1934; además de cuestionarios para el diagnóstico situacional, donde en el enfoque metodológico, se tuvieron presente tres etapas para el desarrollo de la propuesta del plan estratégico.

1. Etapa filosófica: se orienta a la conformación de la imagen futura que se proyecta lograr para el Departamento Docente objeto de investigación, conjuntamente con sus propósitos, principios y valores; así como la misión de esta organización.

2. Etapa analítica: corresponderá al diagnóstico de dicho departamento. 
3. Etapa operativa: será el marco que corresponde al momento estratégico de la organización conformado por un conjunto de políticas, objetivos y estratégias propiamente dichas, las que orientan los planes de acción que conllevará al mejoramiento de las funciones sustantivas y procesos complementarios en dicho departamento.

\section{RESULTADOS}

El plan estratégico del departamento de licenciatura en enfermería; nos sirvió para identificar las principales debilidades y fortalezas que se presentaron, a través de la matriz Foda. El impacto que tuvo en las funciones sustantivas de la universidad hizo que se creará una estructura rígida del diseño curricular que afectaba los niveles de aprendizaje de los estudiantes; sin embargo, con la implementación de un nuevo modelo de plan estratégico hubo un proceso de cambio, lo que constituyo un nuevo paradigma en el régimen académico (MISE 200).

\section{DISCUSIÓN}

El plan estratégico es considerado como un proceso de cambio que permite desarrollar la competitividad presente y futura de quien lo impulsa.

En las universidades debe ser referido a aspiraciones a largo y mediano plazo; tiene la características de ser sistemático, además, de secuencial por su sentido de organización y conducción operacional que tiene lugar por etapas y actúa en base a una realidad concreta, basándose en el desarrollo de la construcción social, además de tener un carácter participativo y lograr llevar a la organización a niveles de excelencia (Flores 1992).

\section{MODELO}

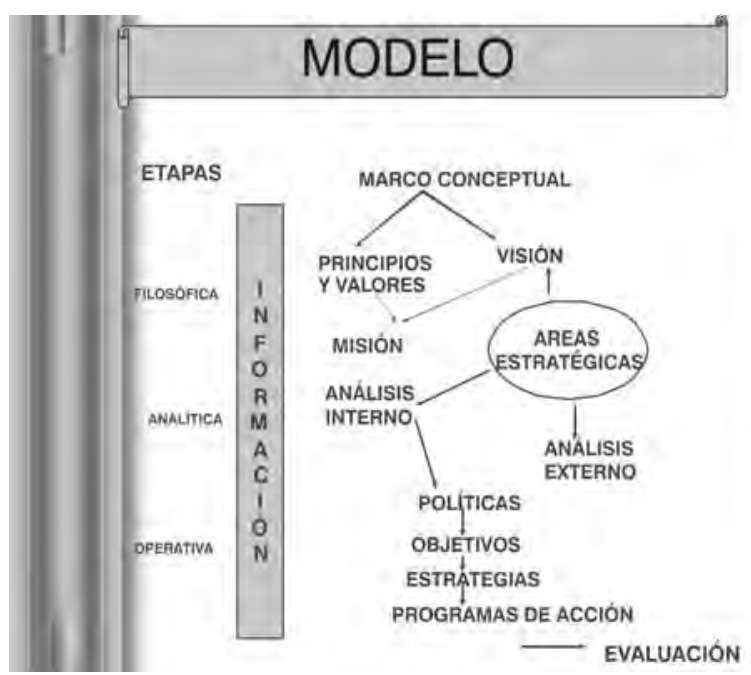

\section{CONCLUSIONES}

La direccionalidad de la propuesta de planificación estratégica que se presentó estuvo orientada hacia el logro de la contextualización del departamento, perfeccionamiento de las funciones de formación de pregrado y posgrado, investigación científica y extensión universitaria: así como, el fortalecimiento de los recursos materiales e incremento de las relaciones de cooperación.

El enfoque metodológico que se utilizó en está investigación fue diseñado para el nivel institucional y facultativo, lo que ha resultado factible para su aplicación en un departamento docente en el cual se desarrolla el proceso de formación.

Los retos a los que se enfrenta el departamento de licenciatura en enfermería para los próximos años, se proyecta en estratégias encaminadas al perfeccionamiento curricular y hacia una mejoría de la organización del proceso docente, fortalecimiento de la integración de los vínculos de investigación con la enseñanza y extensión en la producción de nuevos conocimientos, lo cual debe estar soportada en una infraestructura con una ambientación y estética adecuada.

Dentro de esta proyección estratégica se instrumento la elevación del rendimiento académico del estudiante con un bienestar estudiantil superior al actual.

\section{BIBLIOGRAFÍA CONSULTADA}

1. Acosta Al. La planeación estratégica y la dirección por objetivo Universidad de Matanzas, 1999.

2. Alberdi CR. La formación superior como instrumento para el desarrollo de la enfermería. España, 2000.

3. Imunias JL, Romero B. Evaluación de la enseñanza dirigida en Cuba: una aproximación metodológica. Universidad de la Habana CEPES UNESCO, La Habana. 1994.

4. Álvarez de Zayas C. Fundamentos teóricos de la dirección de proceso docente educativo de la Educación Superior Cubana. Ciudad de la Habana 1998.

5. Bello Fernández N. Logros de la Salud Pública Cubana y formación de Recursos Humanos en Enfermería. Cuba 2000.

6. MINSAP. Teoría y administración de la Salud. Cuba 1998.

7. MINSAP. Anuario estadístico. Cuba 1999.

8. Romero B, Hernández M. Plan piloto de Acción para el cambio y transformación de la educación superior en la Universidad Juan Misael Saracho IESAL / UNESCO. Tarija Bolivia 2000.

9. Romero B. Propuesta de un modelo para gestión de la docencia. Rev Cub Educ Sup 2001;2.

10. Romero B. Propuesta de un modelo organizacional para el Departamento Docente CEPES Universidad de la Habana 2002.

11. World Health Organization. Los servicios de salud en enfermería y partería. Suiza 2000.

12. Zaglul José A. Valores éticos y humanos claves para el desarrollo del próximo milenio. Carta informativa EARTH, Año 5 No. 22, San José Costa Rica 2002. 